Article

\title{
Dynamic Scheduling Based on Predicted Inventory Variation Rate for Public Bicycle System
}

\author{
Liang Gao * , Wei $\mathrm{Xu}$ and Yifeng Duan \\ Key Laboratory of Transport Industry of Big Data Application Technologies for Comprehensive Transport, \\ Beijing Jiaotong University, Beijing 100044, China; xuwei2016@bjtu.edu.cn (W.X.); \\ yifeng_duan@bjtu.edu.cn (Y.D.) \\ * Correspondence: lianggao@bjtu.edu.cn
}

Received: 5 March 2019; Accepted: 27 March 2019; Published: 29 March 2019

\begin{abstract}
To improve efficiency and reduce the total scheduling cost of the public bicycle system (PBS), dynamic scheduling based on the predicted inventory variation rate (DS-PIVR) is proposed. Regarding a station in the PBS as an inventory system, its inventory variation rate during the scheduling period and its inventory rate at the end of the scheduling period were predicted based on the stationary Markov process condition. A mixed integer programming (MIP) model, whose objective is to minimize the total scheduling distance, was established to describe the dynamic scheduling problem (DSP). Results from Boston and Washington D.C. PBSs show that, when compared to the dynamic scheduling based on the rolling horizon (DS-RH), the DS-PIVR method could at most shorten the routing distance by $62.25 \%$ (for Boston) and $74.7 \%$ (for Washington D.C.) among all scheduling areas, and could at most shorten the total routing distance for the whole PBS by $21.06 \%$ (for Boston) and $17.26 \%$ (for Washington D.C.). Moreover, the DS-PIVR method makes the repositioning vehicle journey only once and keeps the inventory rate of each station in balance during the scheduling period. Furthermore, the DS-PIVR method provides a promising reference to improve the operation efficiency by reducing the scheduling cost and the quality of service by satisfying the users' demand in time during the rush hours for the PBS operators.
\end{abstract}

Keywords: public bicycle system; dynamic scheduling; mixed integer programming; inventory variation rate

\section{Introduction}

As a short-distance, carbon-free traffic mode, public bicycles can solve the "last mile" problem of urban public transportation. In recent years, public bicycle systems (PBSs) have been widely used all over the world [1-3]. Studies on PBSs emerge [3,4], including bicycle safety [1] and health [5], modal shift [2], usage patterns [1,4,6], spatial-temporal features of the operations [5,7-9], etc.

The public bicycle systems (PBSs) can be mainly divided into docking and dockless PBSs [10]. The former provides services via docking stations, while the latter realizes the standardized management by setting up electronic fences [10]. The spatial and temporal distribution of travel demand is uneven in PBSs. With the expansion of PBSs and the growth of users, problems in borrow and return processes are gradually emerging $[10,11]$. How to maximize the quality of service and control the operational cost in a rational range has become the primary goal of the PBS operators. Efficiently scheduling the vehicles to rebalance the system is the key to solving this problem [6,12].

To optimize the PBS scheduling process, previous studies [4] suggested that the problem should be divided into three aspects. The first is the forecasting of the rebalancing demand. It mainly includes the mathematical models such as the Markov chain [13], queuing theory [14], etc., and the machine learning models such as neural networks [15], random forest [16,17], gradient boosting regression 
tree (GBRT) [18], etc. The second is the division of the scheduling area. It mainly includes district partition by administrative boundary and clustering by the attributes of stations, such as k-means clustering [19], Gale-Shapley algorithm [20], saving heuristic [21], etc. The third is the repositioning of vehicle routing, which is the core issue of scheduling optimization problems.

The goal of repositioning the vehicle routing could be divided into two categories. One is to reduce the rebalancing cost $[22,23]$, including the total routing distance of the repositioned vehicle, the total execution time of the rebalancing task, and the total number of transferred bicycles. The other is to improve users' satisfaction and simultaneously reduce the rebalancing cost, which is a multi-objective optimization $[24,25]$ considering the trade-off between the operating cost and the service quality. Here, users' satisfaction is commonly quantified by the waiting time, or by the users' satisfaction function based on the fuzzy time window.

There are two main types of PBS scheduling optimization, namely static scheduling, and dynamic scheduling. Static scheduling, often performed at night or in the off-peak period, can ignore the influence of the users borrowing and returning the bicycles during the scheduling procedure. Static scheduling can be simply summarized as the vehicle routing problem (VRP) [26], which can be established by the MIP model $[27,28]$. Dynamic scheduling, often performed during the rush hour, considers the inventory variation of stations during the scheduling procedure. The common methods for dynamic scheduling include the rolling horizon [29] and the time-space network flow [30], both of which divide the scheduling period into discrete time slices. The DS-RH transforms the DSP into the static scheduling problem (SSP) in consecutive time slices [31]. The scheduling scheme of each time slice is passively adjusted with the stage detection, which is essentially the composition of the local optimization scheme. Due to the insufficient information of the prediction, the overall efficiency of the scheduling period in the DS-RH cannot be guaranteed. The time-space network flow method divides the scheduling period into discrete time slices as well and sets several time-space nodes to transform the DSP into the SSP [30]. Since the same station needs to be set as a virtual time-space node in each time slice, the complexity of solving the time-space network flow model will be greatly increased when we meet a large PBS with a long-time scheduling task.

This paper proposes a dynamic scheduling method for the PBS based on the predicted inventory variation rate. By calculating the inventory variation rate of each station, the inventory rate of the station at the end of the scheduling period is actively predicted, and an optimal scheduling scheme for the whole scheduling period is generated. The results prove that compared to the DS-RH method, the DS-PIVR method is more reasonable and effective. DS-PIVR method will provide a promising reference to improve the operation efficiency and the quality of service of a PBS during the rush hours.

The remainder of this paper is organized as follows. Section 2 introduces the properties of a station of a PBS. Dynamic scheduling based on the predicted inventory variation rate is proposed in Section 3. Then, Section 4 shows the case of the Boston PBS to test the DS-DIVR method. The last section discusses the results compared with previous studies and makes recommendations for future research directions.

\section{Properties of a Station}

A station, which provides a borrow and return service via bicycle docks, can be regarded as an inventory system $[5,28]$. We define the properties of a station and provide the related calculation methods.

\subsection{Inventory Variation Rate}

During the scheduling period $T$, the cumulative number of borrowed and returned bicycles at a station $i$ is denoted as $B_{i}$ and $R_{i}$, respectively. The average borrowing and returning rate of a station $i$ are defined as $\lambda_{i}=B_{i} / T$ and $\mu_{i}=R_{i} / T$, respectively. Then the inventory variation rate of a station $i$ is $\delta_{i}=\lambda_{i}-\mu_{i}$, which depicts the borrow-return trend of a station. The larger the positive value of $\delta_{i}$, the stronger the borrowing demand; the larger the negative value of $\delta_{i}$, the stronger the return demand. 
According to the historical operation data of the Boston PBS, the predicted inventory variation rate of a station could be calculated as the following steps.

First, the travel demand OD matrix is obtained in the period $T$ as $\boldsymbol{F}=\left(f_{i j}\right)_{n \cdot n}$, where $f_{i j}$ is the travel amount from station $i$ to station $j$. The borrow transfer probability matrix is obtained:

$$
\boldsymbol{P}=\left(p_{i j}\right)_{n \cdot n}=\left(\frac{f_{i j}}{\sum_{k=1}^{n} f_{i k}}\right)_{n \cdot n}
$$

where $p_{i j}$ indicates the probability of the bicycles borrowed from station $i$ and returned to station $j$. Accordingly, the return transfer probability matrix is obtained:

$$
Q=\left(q_{i j}\right)_{n \cdot n}=\left(\frac{f_{i j}}{\sum_{k=1}^{n} f_{k j}}\right)_{n \cdot n}
$$

where $q_{i j}$ indicates the probability of the bicycles borrowed from station $i$ and returned to station $j$.

Secondly, assuming that the borrow-return process is a stationary Markov process [4]. According to the stationary distribution of the Markov process [32], we obtain:

$$
\left\{\begin{array}{c}
\varphi_{b}=\varphi_{b} \cdot P \\
\varphi_{r}=Q \cdot \varphi_{r}
\end{array}\right.
$$

where $\varphi_{b}$ and $\varphi_{r}$ indicate the stationary probability vector of the borrow and return in the period $T$, respectively.

Then, the cumulative number of borrowed and returned bicycles are obtained as follows:

$$
\left\{\begin{array}{l}
\boldsymbol{B}=\boldsymbol{\varphi}_{b} \cdot \bar{h} \\
\boldsymbol{R}=\bar{h} \cdot \boldsymbol{\varphi}_{r}
\end{array}\right.
$$

where $\boldsymbol{B}$ and $\boldsymbol{R}$ are vectors come up with the cumulative number of borrowed and returned bicycles of each station in the scheduling period $T, \bar{h}$ indicates the average hourly trips in the same period calculated by using historical trip data.

Finally, the inventory variation rate is obtained:

$$
\delta_{i}=\left(B_{i}-R_{i}\right) / T
$$

\subsection{Predicted Inventory Rate}

The number of docks at station $i$ is defined as the station capacity $C_{i}$. The number of bicycles stored at station $i$ at moment $t$ is defined as inventory $V_{i}(t)$. The ratio of inventory to capacity is defined as the inventory rate $S_{i}(t)=\frac{V_{i}(t)}{C_{i}}$.

At the beginning moment $t_{0}$ of the time period $T$, the initial inventory rate of station $i$ is defined as $S_{i}\left(t_{0}\right)=\frac{V_{i}\left(t_{0}\right)}{C_{i}}$, then the predicted inventory rate of station $i$ at the moment $\left(t_{0}+T\right)$ will be

$$
\widetilde{S}_{i}\left(t_{0}+T\right)=S_{i}\left(t_{0}\right)-\delta_{i} \cdot T / C_{i} .
$$

If either $S_{i}\left(t_{0}\right)$ or $\widetilde{S}_{i}\left(t_{0}+T\right)$ exceeds the reasonable interval $\left[S_{\min }, S_{\max }\right]$, then station $i$ will be rebalanced during the time period $T$. Let $t_{i}$ represent the time that the repositioning vehicle 
arrives at station $i$. Thus, the optimization object is to keep the inventory rate and always stay in a reasonable interval

$$
S_{\min }<S_{i}(t)<S_{\max }, t \in\left[t_{i}, t_{0}+T\right]
$$

\subsection{Rebalancing Demand}

The target inventory $\hat{V}_{i}\left(t_{i}\right)$ of station $i$ at $t_{i}$ is calculated as:

$$
\hat{V}_{i}\left(t_{i}\right)=\left[\left(\frac{\left(S_{\max }-S_{\min }\right)}{\pi} \cdot \tan ^{-1}\left(\delta_{i} \cdot\left(t_{0}+T-t_{i}\right)\right)+\frac{S_{\max }+S_{\min }}{2}\right) \cdot C_{i}\right]
$$

The rebalancing demand of station $i$

$$
q_{i}=\left[\hat{V}_{i}\left(t_{i}\right)-\left(V_{i}\left(t_{0}\right)-\delta_{i} \cdot\left(t_{i}-t_{0}\right)\right)\right]
$$

where $V_{i}\left(t_{0}\right)$ is the initial inventory. If $q_{i}>0$, it indicates the delivery requirement, else if $q_{i}<0$, it indicates the pickup requirement.

\subsection{Latest Arrival Time}

To avoid an empty or full situation, the repositioning of vehicles is limited to the latest arrival time of station $i$, which is defined as

$$
D_{i}= \begin{cases}\min \left(t_{0}+\frac{V_{i}\left(t_{0}\right)}{\delta_{i}}, t_{0}+T\right) & \delta_{i}>0 \\ t_{0}+T & \delta_{i}=0 \\ \min \left(t_{0}+\frac{V_{i}\left(t_{0}\right)-C_{i}}{\delta_{i}}, t_{0}+T\right) & \delta_{i}<0\end{cases}
$$

\section{Methodology}

To avoid the lag of scheduling, a regional and phased strategy is adopted. In this paper, a dynamic scheduling optimization method based on predicted inventory variation rate $\delta_{i}$, is studied in a given scheduling period and scheduling area. For each scheduling area, we assigned one depot and one repositioning vehicle.

\section{Scheduling Scheme}

In a given scheduling area, the scheduling scheme is shown in Figure 1. The generating process is:

Step 1: Predict the station inventory rate at the end of the scheduling period $\widetilde{S}_{i}\left(t_{0}+T\right)$.

Step 2: Identify the set of stations with rebalancing requirements. The initial inventory rate $S_{i}\left(t_{0}\right)$ at each station, the predicted inventory rate at the end of the scheduling period $\widetilde{S}_{i}\left(t_{0}+T\right)$ and the reasonable inventory rate interval $\left[S_{\min }, S_{\max }\right]$ are compared to determine the stations with rebalancing demands.

Step 3: Calculate the latest arrival time $D_{i}$.

Step 4: Establish the scheduling optimization model by minimizing the routing distance of the repositioned vehicle.

Step 5: Solve the optimization model and obtain the scheduling scheme.

The latest arrival time is used to describe the urgency of the rebalancing requirement of a station. According to the service order of the repositioning vehicle, the rebalancing demand of each station is dynamically calculated. According to the initial inventory rate and the predicted inventory rate, it is judged whether a station should be rebalanced. 


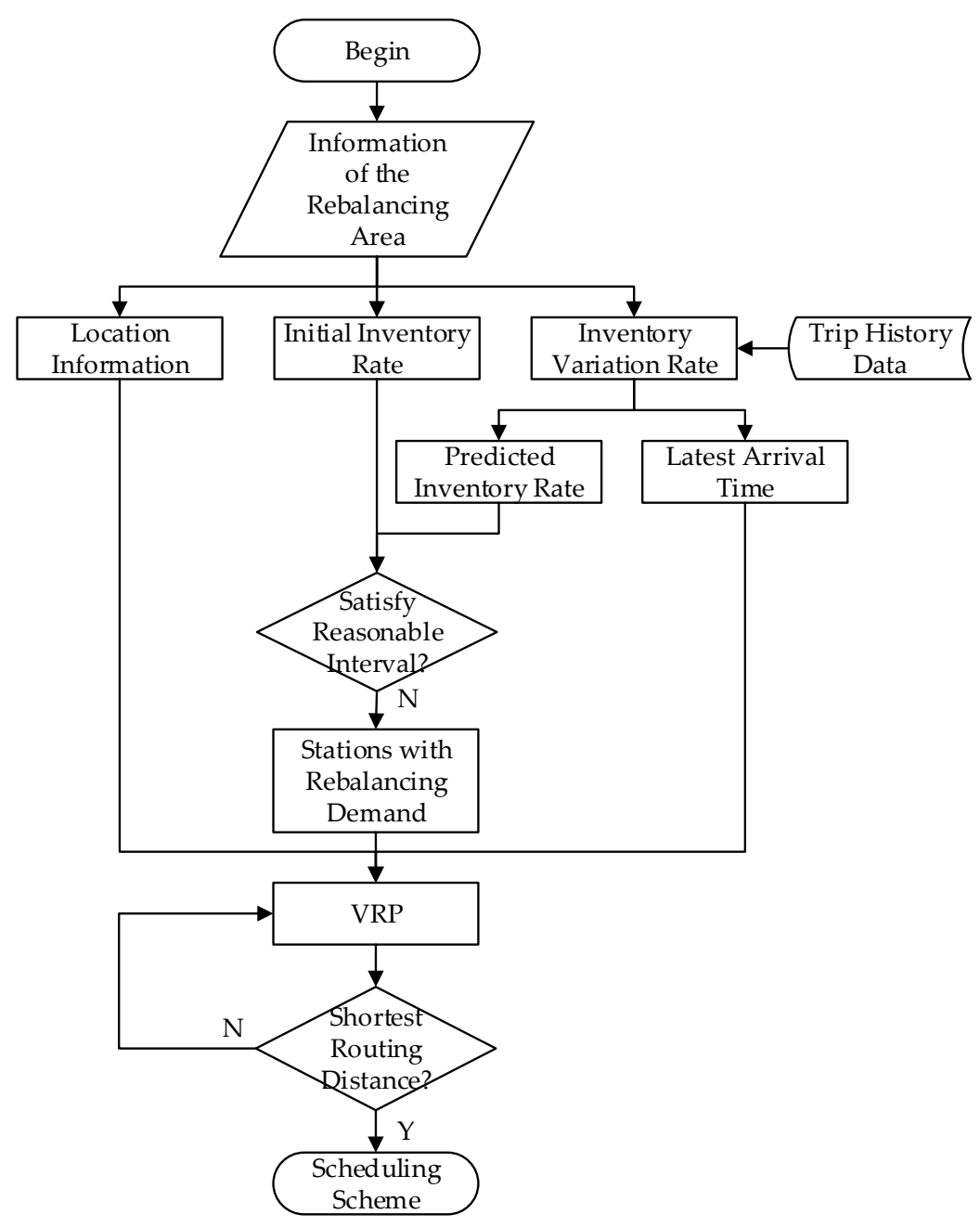

Figure 1. Generating process of scheduling scheme.

The heart of the scheduling process is a vehicle routing problem (VRP). An MIP model $[13,27]$ is established to describe the scheduling procedure as

$$
\operatorname{Min} \mathrm{Z}=\sum_{i \neq j \in N_{0}} d_{i j} x_{i j}
$$

s.t.

$$
\begin{gathered}
t_{j} \geq t_{i}+t_{w}\left|q_{i}\right|+t_{i j} x_{i j}+P\left(x_{i j}-1\right) \quad \forall i \neq j \in N_{0} \\
\sum_{j \neq i \in N_{0}} x_{j i}=\sum_{j \neq i \in N_{0}} x_{i j}=1 \quad \forall i \in N_{0} \\
\sum_{i \neq j \in N_{0}} t_{i j} x_{i j}+\sum_{i \in N} t_{w}\left|q_{i}\right| \leq T \\
\sum_{i \neq j \in S} x_{i j} \leq|S|-1 \quad \forall S \subseteq N, 2 \leq|S| \leq|N| \\
0 \leq Q_{i}-\sum_{j \neq i \in N_{0}} x_{i j} q_{j} \leq Q \quad \forall i \in N_{0} \\
0 \leq Q_{0} \leq Q \\
x_{i j} \in\{0,1\} \quad \forall i \neq j \in N_{0} \\
t_{0} \leq t_{i} \leq D_{i} \quad \forall i \in N_{0}
\end{gathered}
$$


The objective function (11) minimizes the routing distance of repositioning the vehicle, Constraints (12) limit visiting times of stations, which ensure the minimal time required for repositioning the vehicle to travel from station $i$ to station $j$. Constraints (13) take care of flow conservation, which ensures each station with rebalancing demand visited only once by the repositioning vehicle, and it means that the vehicle starts from the depot and finally returns to the depot. Constraints (14) limit the total routing times of repositioning vehicles plus total loading and unloading times to the total time of the scheduling period. Constraints (15) are sub-tour elimination constraints which are similar to those of Miller et al. [33]. Constraints (16) ensure that the vehicle inventory remains non-negative and within capacity after leaving station $i$. Constraint (17) is the limit on the initial inventory of repositioning vehicles. Constraints (18) are binary constraints. Constraints (19) take care of visiting time of each station within the latest arrival time. All parameters are listed, and explained in Table 1.

Table 1. Parameters used in the model.

\begin{tabular}{cc}
\hline$N$ & Station sets that need to be rebalanced \\
\hline$N_{0}$ & The union of the set of stations that need to be rebalanced and the depot, which is marked as 0 \\
\hline$q_{i}$ & $\begin{array}{r}\text { The rebalancing demand of station } i, \text { a positive value indicates the delivery requirement, } \\
\text { while a negative value indicates the pickup requirement. }\end{array}$ \\
\hline$Q_{0}$ & The initial load of the repositioning vehicle \\
\hline$Q_{i}$ & Number of bicycles loaded after repositioning vehicle leaving the station $i$ \\
\hline$Q$ & The load limit of the repositioning vehicle, up to 30 bicycles \\
\hline$T$ & Scheduling time period, take $1 \mathrm{~h}$ \\
\hline$t_{0}$ & Initial time of scheduling \\
\hline$t_{i j}$ & Pistance from station $i$ to station $j$ \\
\hline$d_{i j}$ & The latest arrival time of station $i$ \\
\hline$D_{i}$ & The time when the repositioning vehicle arrives at station $i$ \\
\hline$P$ & Time required to load or unload a bicycle from the repositioning vehicle at station $i$, take $20 \mathrm{~s}$ \\
\hline$x_{i j}$ & Decision variable, which equals 1 if the vehicle travels directly from station $i$ to station $j$, and 0 otherwise \\
\hline$t_{i}$ &
\end{tabular}

\section{Validation of DS-PIVR}

Take the Boston PBS at the evening rush hour (17:00-18:00) of the weekday as an example to verify the dynamic scheduling based on the predicted inventory variation rate (DS-PIVR).

According to the maximum spanning star [28], the workload constraint of the scheduling area is judged. With the scanning algorithm $[34]$ and the k-means $(K \equiv 2)$ clustering algorithm, the scheduling area of the Boston PBS is divided, and 17 scheduling areas are obtained (see Figure 2). Table 2 lists related station information of the 17 scheduling areas.

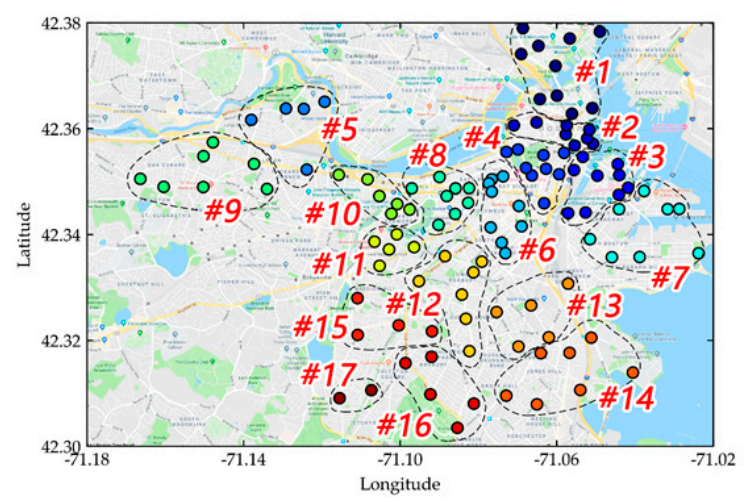

Figure 2. The results of the scheduling area division. The number in the figure is the area ID. 
Table 2. Station information of 17 scheduling areas.

\begin{tabular}{ccc}
\hline Area ID & Number of Stations & Station ID \\
\hline$\# 1$ & 10 & $40,47,85,129,38,98,94,190,169,171$ \\
$\# 2$ & 8 & $43,48,151,20,23,44,6,60$ \\
$\# 3$ & 9 & $31,24,7,64,192,22,63,150,65$ \\
$\# 4$ & 9 & $59,49,81,35,42,54,120,58,152$ \\
$\# 5$ & 5 & $17,29,149,15,41$ \\
$\# 6$ & 9 & $50,134,36,16,4,25,26,39,13$ \\
$\# 7$ & 8 & $21,55,61,52,53,33,5,46$ \\
$\# 8$ & 8 & $37,8,207,66,175,208,174$ \\
$\# 9$ & 7 & $45,122,32,19,10,9$ \\
$\# 10$ & 6 & $160,3,11,14,30$ \\
$\# 11$ & 5 & $56,200,51,12,27,196,204$ \\
$\# 12$ & 7 & $113,138,106,199,128$ \\
$\# 13$ & 5 & $167,130,93,203,205,173,92$ \\
$\# 14$ & 7 & $197,131,159,125$ \\
$\# 15$ & 4 & $201,126,170,202,162$ \\
$\# 16$ & 5 & 133,124 \\
$\# 17$ & 2 &
\end{tabular}

Here, taking Area \#3 as an example, the scheduling scheme is shown. The status of all stations in Area \#3 is shown in Table 3. The simulated annealing (SA) algorithm is used to solve the scheduling problem. Figure 3 shows the DS-PIVR and DS-RH schemes. The routing distance of the DS-PIVR and DS-RH scheduling schemes are $3822.97 \mathrm{~m}$ and $5666.36 \mathrm{~m}$, respectively.

Table 3. Status of stations in Area \#3 before rebalancing.

\begin{tabular}{cccc}
\hline Station ID & Station Capacity & Initial Inventory & Inventory Variation Rate \\
\hline 31 & 15 & 13 & -1 \\
24 & 19 & 5 & 2 \\
7 & 15 & 6 & 1 \\
64 & 19 & 14 & -1 \\
192 & 19 & 2 & -1 \\
22 & 46 & 7 & -4 \\
63 & 15 & 8 & 0 \\
150 & 19 & 2 & -1 \\
65 & 19 & 14 & -3 \\
\hline
\end{tabular}
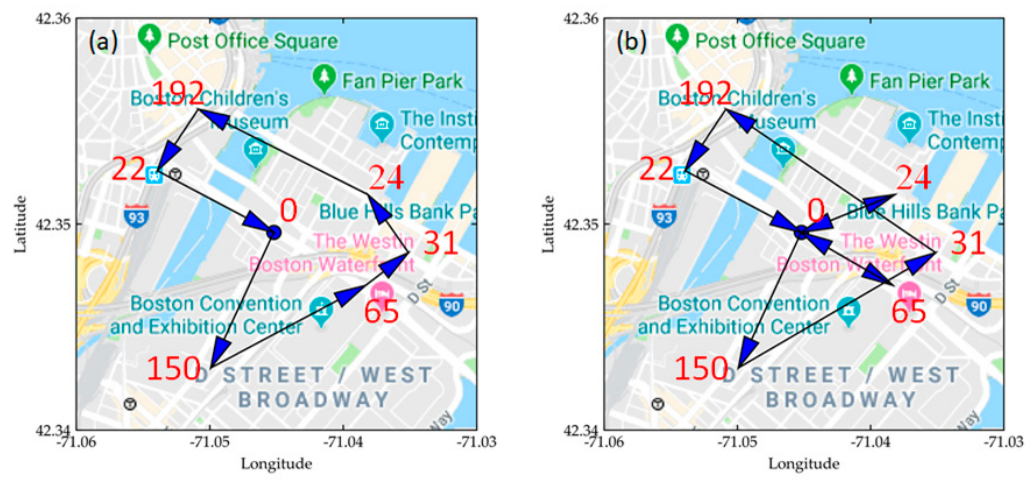

Figure 3. (a) DS-PIVR and (b) DS-RH scheme of Area \#3. The numbers in the figures are the station ID, and 0 represents the depot. The scheduling route of DS-PIVR scheme is: $0 \rightarrow 150 \rightarrow 65 \rightarrow 31 \rightarrow 24 \rightarrow 192 \rightarrow 22 \rightarrow 0$; and that of DS-RH scheme is: $0 \rightarrow 150 \rightarrow 31 \rightarrow 192 \rightarrow 22 \rightarrow 0 \rightarrow 65 \rightarrow 0 \rightarrow 24 \rightarrow 0$. 
Figure 4 shows the complete scheduling scheme of the DS-PIVR and DS-RH methods for the whole Boston PBS under the given initial inventory rate setting. The DS-PIVR method can at most shorten the routing distance of the repositioning vehicle by up to $36.55 \%$, and the total routing distance of the system can be reduced by $18.59 \%$, compared to the DS-RH method (see Table 4).
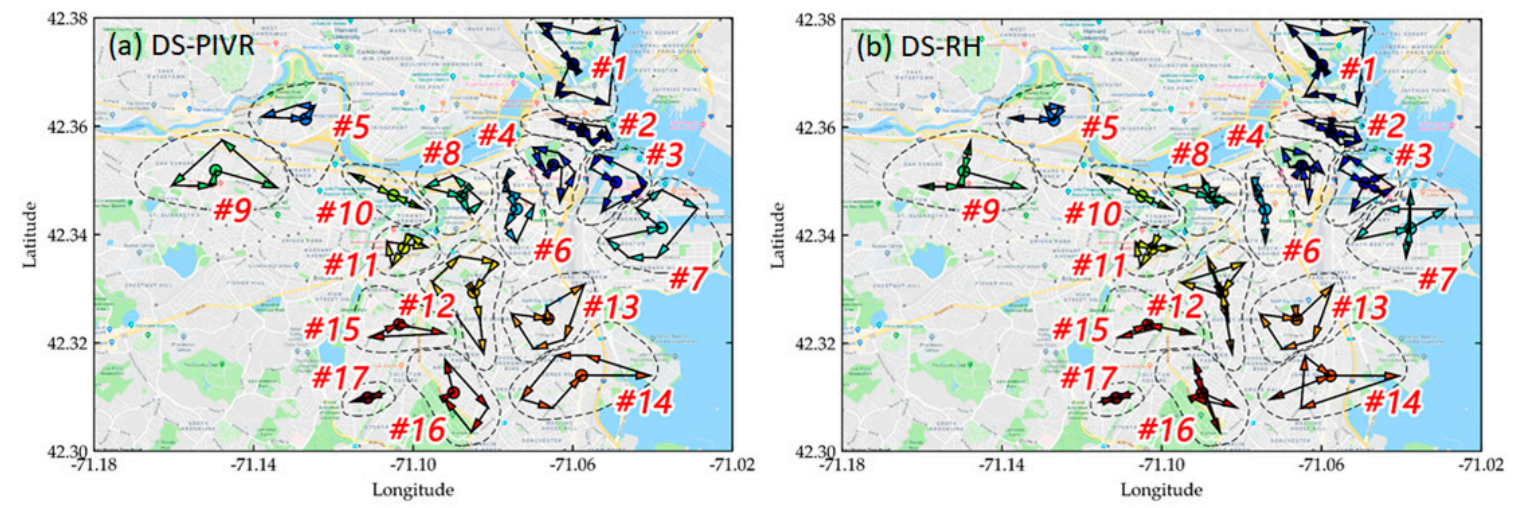

Figure 4. (a) DS-PIVR and (b) DS-RH schemes for the whole Boston PBS.

Table 4. Comparison of scheduling schemes.

\begin{tabular}{|c|c|c|c|c|c|}
\hline \multirow{2}{*}{ Area ID } & \multicolumn{2}{|l|}{ DS-PIVR } & \multicolumn{2}{|l|}{ DS-RH } & \multirow{2}{*}{$\begin{array}{c}\text { Reduction } \\
\text { Ratio (\%) }\end{array}$} \\
\hline & Route & Distance & Route & Distance & \\
\hline$\# 1$ & $0 \rightarrow 98 \rightarrow 190 \rightarrow 38 \rightarrow 40 \rightarrow 85 \rightarrow 129 \rightarrow 169 \rightarrow 94 \rightarrow 0$ & 6345.50 & $0 \rightarrow 98 \rightarrow 169 \rightarrow 129 \rightarrow 85 \rightarrow 40 \rightarrow 38 \rightarrow 190 \rightarrow 0 \rightarrow 94 \rightarrow 0$ & 7468.62 & 15.04 \\
\hline \#2 & $0 \rightarrow 44 \rightarrow 48 \rightarrow 151 \rightarrow 43 \rightarrow 20 \rightarrow 6 \rightarrow 0$ & 3180.53 & $0 \rightarrow 48 \rightarrow 43 \rightarrow 20 \rightarrow 6 \rightarrow 0 \rightarrow 151 \rightarrow 0 \rightarrow 44 \rightarrow 0$ & 4045.74 & 21.39 \\
\hline \#4 & $0 \rightarrow 81 \rightarrow 152 \rightarrow 59 \rightarrow 54 \rightarrow 120 \rightarrow 42 \rightarrow 49 \rightarrow 0$ & 3502.15 & $0 \rightarrow 42 \rightarrow 120 \rightarrow 54 \rightarrow 59 \rightarrow 0 \rightarrow 49 \rightarrow 0 \rightarrow 81 \rightarrow 152 \rightarrow 0$ & 4346.82 & 19.43 \\
\hline$\# 5$ & $0 \rightarrow 29 \rightarrow 149 \rightarrow 15 \rightarrow 0$ & 2386.70 & $0 \rightarrow 15 \rightarrow 0 \rightarrow 149 \rightarrow 29 \rightarrow 0$ & 2861.84 & 16.60 \\
\hline \#6 & $0 \rightarrow 25 \rightarrow 39 \rightarrow 4 \rightarrow 16 \rightarrow 134 \rightarrow 36 \rightarrow 0$ & 3300.59 & $0 \rightarrow 134 \rightarrow 16 \rightarrow 0 \rightarrow 39 \rightarrow 0 \rightarrow 36 \rightarrow 0$ & 3962.96 & 16.71 \\
\hline \#9 & $0 \rightarrow 66 \rightarrow 37 \rightarrow 174 \rightarrow 175 \rightarrow 0$ & 5368.51 & $0 \rightarrow 66 \rightarrow 174 \rightarrow 175 \rightarrow 0 \rightarrow 37 \rightarrow 0$ & 5924.42 & 9.38 \\
\hline$\# 10$ & $0 \rightarrow 9 \rightarrow 19 \rightarrow 45 \rightarrow 0$ & 3316.31 & $0 \rightarrow 45 \rightarrow 19 \rightarrow 9 \rightarrow 0$ & 3316.31 & 0.00 \\
\hline \#11 & $0 \rightarrow 30 \rightarrow 11 \rightarrow 160 \rightarrow 3 \rightarrow 0$ & 2564.48 & $0 \rightarrow 30 \rightarrow 11 \rightarrow 0 \rightarrow 3 \rightarrow 0 \rightarrow 160 \rightarrow 0$ & 2923.58 & 12.28 \\
\hline$\# 12$ & $0 \rightarrow 56 \rightarrow 204 \rightarrow 196 \rightarrow 27 \rightarrow 12 \rightarrow 51 \rightarrow 200 \rightarrow 0$ & 5419.80 & $0 \rightarrow 56 \rightarrow 27 \rightarrow 51 \rightarrow 0 \rightarrow 12 \rightarrow 204 \rightarrow 0 \rightarrow 196 \rightarrow 0$ & 8542.36 & 36.55 \\
\hline$\# 13$ & $0 \rightarrow 138 \rightarrow 113 \rightarrow 128 \rightarrow 199 \rightarrow 106 \rightarrow 0$ & 4635.72 & $0 \rightarrow 113 \rightarrow 128 \rightarrow 199 \rightarrow 106 \rightarrow 0 \rightarrow 138 \rightarrow 0$ & 5003.24 & 7.35 \\
\hline$\# 14$ & $0 \rightarrow 92 \rightarrow 167 \rightarrow 130 \rightarrow 203 \rightarrow 205 \rightarrow 0$ & 6132.41 & $0 \rightarrow 92 \rightarrow 205 \rightarrow 130 \rightarrow 0 \rightarrow 203 \rightarrow 0$ & 7942.72 & 22.79 \\
\hline
\end{tabular}

To comprehensively validate the DS-PIVR method, we employ the validation process of previous works $[13,27,28,35,36]$, and numerically generate 50 independent settings for each station of Boston PBS and Washington D.C. PBS, respectively. Table 5 summarizes our computational results for Boston and Washington D.C. PBSs. Compared to DS-RH method, the best found MIP solution of the DS-PIVR method among all scheduling areas can shorten the routing distance of the repositioning vehicle by $62.25 \%$ (for Boston) and $74.7 \%$ (for Washington D.C.), and the best found MIP solution for the whole PBS can shorten the total routing distance by $21.06 \%$ (for Boston) and $17.26 \%$ (for Washington D.C.). In addition, 17 settings for Boston and 14 settings for Washington D.C. (out of 50) did not yield an MIP solution for the DS-PIVR method or DS-RH method.

Table 5. Results for Boston and Washington D.C. PBSs.

\begin{tabular}{|c|c|c|c|c|c|}
\hline \multirow[t]{2}{*}{ Case } & \multirow{2}{*}{$\begin{array}{l}\text { Number of } \\
\text { Settings with } \\
\text { MIP Solution }\end{array}$} & \multicolumn{2}{|c|}{$\begin{array}{c}\text { Reduction Ratio Among } \\
\text { All Areas }\end{array}$} & \multicolumn{2}{|c|}{$\begin{array}{c}\text { Reduction Ratio for the } \\
\text { Whole PBS }\end{array}$} \\
\hline & & Best Found & Average & Best Found & Average \\
\hline Boston & 33 & $62.25 \%$ & $13.68 \%$ & $21.06 \%$ & $16.93 \%$ \\
\hline Washington DC & 36 & $74.70 \%$ & $10.66 \%$ & $17.26 \%$ & $14.37 \%$ \\
\hline
\end{tabular}




\section{Discussion}

This paper proposes a dynamic scheduling method based on the predicted inventory variation rate for the rebalancing of a PBS. The bicycle station is regarded as an inventory system. The rebalancing demand of the stations and the scheduling route of the repositioning vehicle are considered simultaneously. Based on the historical trip data and the theory of the stationary Markov process, the inventory variation rate of each station during the whole scheduling period is predicted. The station inventory rate at the end of the scheduling period and the latest arrival time constraint for each station are calculated. The routing distance of the repositioning vehicle is set as the objective function, and the binary nonlinear MIP model is solved to obtain an optimized scheduling scheme. The results prove that the proposed method significantly improves the scheduling efficiency and reduces the scheduling cost, compared to the DS-RH method. The scheduling scheme generated by the proposed method ensures that the repositioning of the vehicles make the journey only once to keep all stations rebalanced for each given area. Furthermore, the DS-PIVR method is applicable to the dynamic scheduling situation during daily rush hours and can be used as a promising reference for the public bicycle operators to obtain a reasonable scheduling scheme.

Meanwhile, in the proposed model, the inventory variation rate in a scheduling period is predicted based on the historical operation data. The accuracy of the prediction will be determined by the selection of the prediction model. Therefore, how to select a more accurate and more adaptable prediction model is a good question to make the DS-PIVR method more practical.

The proposed model takes the area division as a precondition, which is originated from two considerations. On the one hand, for the bicycle dispatchers, the constant scheduling area is the more reasonable and operable task. If the scheduling area varies by time, then the bicycle dispatchers have to pay more attention to be familiar with the routes and the area, which will increase the complexity and decrease the efficiency of dispatching process. On the other hand, the scheduling area is independent with each other, i.e., the scheduling vehicle can only cruise in its assigned area. The purpose is to ensure that the scheduling scheme meets the scheduling requirements with time constraints and operability. Therefore, how to divide the scheduling areas simultaneously meeting the variation of scheduling requirements in a different scheduling period and keeping the operability for the practitioners is one of the important future works.

The compilation of the scheduling scheme includes a VRP solution process. To simplify the whole rebalancing problem, the traffic condition on the roads was not taken into consideration in the proposed model. Considering the traffic condition, and even considering the real-time traffic condition via an intelligent transport system, will improve the practicability of the proposed model a lot.

Furthermore, to our knowledge, there are two more future directions to extend our work. One is to take the state before the current scheduling period into consideration to improve the accuracy of the prediction procedure. The other is to remove the constant assumption about the inventory variation rate during the scheduling period, which will make the future works more practical for the real PBS. Both of them will provide a promising way to reduce the scheduling cost further, and improve the quality of the service (QoS) of a real PBS.

\section{Conclusions}

Based on the predicted inventory variation rate, a dynamic scheduling method called a DS-PIVR method for the rebalancing of a PBS is proposed. Results of extensive experiments prove that the proposed method could at most shorten the routing distance by $62.25 \%$ (for Boston) and $74.7 \%$ (for Washington D.C.) among all scheduling areas, and could at most shorten the total routing distance for the whole PBS by $21.06 \%$ (for Boston) and $17.26 \%$ (for Washington D.C.). It is believed that the DS-PIVR method provides a promising reference to improve the operation efficiency by reducing the scheduling cost and the quality of the service by satisfying the users' demand in time during the rush hours for the PBS operating companies. 
Author Contributions: L.G. conceived and designed the experiments; L.G. and W.X. prepared the data and wrote the manuscript; W.X. analyzed the data and performed the experiments in this article; Y.D. edited the manuscript.

Funding: This research was funded by the National Natural Science Foundation of China (No.91646124, No.71571017, No.71621001, and No. 91746201).

Acknowledgments: The authors thank for support from the Fundamental Research Funds for the Central Universities (2018JBM026).

Conflicts of Interest: The authors declare no conflict of interest.

\section{References}

1. Martin, E.; Cohen, A.; Botha, J.; Shaheen, S. Bikesharing and Bicycle Safety. Mineta Transportation Institute Publications. Available online: http://transweb.sjsu.edu/research/bikesharing-and-bicycle-safety (accessed on 18 March 2019).

2. Shaheen, S.; Martin, E.; Cohen, A. Public Bikesharing and Modal Shift Behavior: A Comparative Study of Early Bikesharing Systems in North America. Int. J. Transp. 2013, 1, 35-54. [CrossRef]

3. Pucher, J.; Buehler, R. Cycling towards a more sustainable transport future. Transp. Rev. 2017, 37, 689-694. [CrossRef]

4. Fishman, E. Bikeshare: A Review of Recent Literature. Transp. Rev. 2016, 36, 92-113. [CrossRef]

5. Lu, T.; Mondschein, A.; Buehler, R.; Hankey, S. Adding temporal information to direct-demand models: Hourly estimation of bicycle and pedestrian traffic in Blacksburg, VA. Transp. Res. Part D 2018, 63, 244-260. [CrossRef]

6. Faghih-Imani, A.; Hampshire, R.; Marla, L.; Eluru, N. An empirical analysis of bike sharing usage and rebalancing: Evidence from Barcelona and Seville. Transp. Res. Part A 2017, 97, 177-191. [CrossRef]

7. Wergin, J.; Buehler, R. Where Do Bikeshare Bikes Actually Go? Analysis of Capital Bikeshare Trips with GPS Data. Transp. Res. Rec. 2017, 2662, 12-21. [CrossRef]

8. Hankey, S.; Lu, T.; Mondschein, A.; Buehler, R. Spatial models of active travel in small communities: Merging the goals of traffic monitoring and direct-demand modeling. J. Transp. Health Part B 2017, 7, 149-159. [CrossRef]

9. Lu, T.; Buehler, R.; Mondschein, A.; Hankey, S. Designing a Bicycle and Pedestrian Traffic Monitoring Program to Estimate Annual Average Daily Traffic in a Small Rural College Town. Transp. Res. Part D 2017, 53, 193-204. [CrossRef]

10. Shaheen, S.; Guzman, S.; Zhang, H. Bikesharing in Europe, the Americas, and Asia: Past, Present, and Future. Transp. Res. Rec. 2011, 2143, 159-167. [CrossRef]

11. Cagliero, L.; Cerquitelli, T.; Chiusano, S.; Garza, P.; Ricupero, G.; Baralis, E. Characterizing Situations of Dock Overload in Bicycle Sharing Stations. Appl. Sci. 2018, 8, 2521. [CrossRef]

12. Li, L.; Shan, M. Bidirectional Incentive Model for Bicycle Redistribution of a Bicycle Sharing System during Rush Hour. Sustainability 2016, 8, 1299. [CrossRef]

13. Zhou, Y.; Wang, L.; Zhong, R.; Tan, Y. A Markov Chain Based Demand Prediction Model for Stations in Bike Sharing Systems. Math. Prob. Eng. 2016, 2016, 8028714. [CrossRef]

14. Raviv, T.; Kolka, O. Optimal Inventory Management of a Bike-sharing Station. IIE Trans. 2013, 45, 1077-1093. [CrossRef]

15. Xu, C.; Ji, J.; Liu, P. The Station-free Sharing Bike Demand forecasting with a Deep Learning Approach and Large-scale Datasets. Transp. Res. Part C 2018, 95, 47-60. [CrossRef]

16. Feng, Y.; Wang, S. A Forecast for Bicycle Rental Demand based on Random Forests and Multiple Linear Regression. In Proceedings of the IEEE/ACIS International Conference on Computer and Information Science, Wuhan, China, 24-26 May 2017. [CrossRef]

17. Ashqar, H.I.; Elhenawy, M.; Almannaa, M.H.; Ghanem, A.; Rakha, H.A.; House, L. Modeling Bike Availability in a Bike-Sharing System Using Machine Learning. In Proceedings of the 5th IEEE International Conference on Models and Technologies for Intelligent Transportation Systems, Naples, Italy, 26-28 June 2017. [CrossRef]

18. Jiang, J.; Lin, F.; Fan, J.; Lv, H.; Wu, J. A Destination Prediction Network Based on Spatiotemporal Data for Bike-Sharing. Complexity 2019, 2019, 7643905. [CrossRef]

19. Dong, H.; Shi, C.; Chen, N.; Liu, D. Clustering Division of Public Bicycle Scheduling Regional Based on Association Rules. Bullet. Sci. Techn. 2013, 29, 209-212. [CrossRef] 
20. Almannaa, M.H.; Elhenawy, M.; Ghanem, A.; Ashqar, H.I.; Rakha, H.A. Network-Wide Bike Availability Clustering Using the College Admission Algorithm: A Case Study of San Francisco Bay Area. In Proceedings of the 5th IEEE International Conference on Models and Technologies for Intelligent Transportation Systems, Naples, Italy, 26-28 June 2017. [CrossRef]

21. Forma, I.A.; Raviv, T.; Tzur, M. A 3-step Math Heuristic for the Static Repositioning Problem in Bike-sharing Systems. Transp. Res. Part B 2015, 71, 230-247. [CrossRef]

22. Dell'Amico, M.; Hadjicostantinou, E.; Iori, M.; Novellani, S. The Bike Sharing Rebalancing Problem: Mathematical Formulations and Benchmark Instances. Omega 2014, 45, 7-19. [CrossRef]

23. Erdogan, G.; Battarra, M.; Calvo, R.W. An Exact Algorithm for the Static Rebalancing Problem arising in Bicycle Sharing Systems. Eur. J. Oper. Res. 2015, 245, 667-679. [CrossRef]

24. Li, Y.; Szeto, W.Y.; Long, J.; Shui, C.S. A Multiple Type Bike Repositioning Problem. Transp. Res Part B 2016, 90, 263-278. [CrossRef]

25. Szeto, W.; Liu, Y.; Ho, S. Chemical Reaction Optimization for Solving a Static Bike Repositioning Problem. Transp. Res. Part D 2016, 47, 104-135. [CrossRef]

26. Braekers, K.; Ramaekers, K.; Nieuwenhuyse, I.V. The Vehicle Routing Problem: State of the Art Classification and Review. Comput. Ind. Eng. 2016, 99, 300-313. [CrossRef]

27. Raviv, T.; Tzur, M.; Forma, I.A. Static Repositioning in a Bike-sharing System: Models and Solution Approaches. Eur. J. Transp. Logist. 2013, 2, 187-229. [CrossRef]

28. Schuijbroek, J.; Hampshire, R.C.; Hoeve, W.J. Inventory Rebalancing and Vehicle Routing in Bike Sharing Systems. Eur. J. Oper. Res. 2017, 257, 992-1004. [CrossRef]

29. Shui, C.S.; Szeto, W.Y. Dynamic Green Bike Repositioning Problem-A Hybrid Rolling Horizon Artificial Bee Colony Algorithm Approach. Transp. Res. Part D 2018, 60, 119-136. [CrossRef]

30. Zhang, D.; Yu, C.; Desai, J.; Lau, H.Y.K.; Srivathsan, S. A Time-space Network Flow Approach to Dynamic Repositioning in Bicycle Sharing Systems. Transp. Res. Part B 2017, 103, 188-207. [CrossRef]

31. Yu, K.; Yang, J. MILP Model and a Rolling Horizon Algorithm for Crane Scheduling in a Hybrid Storage Container Terminal. Math. Prob. Eng. 2019, 2019, 4739376. [CrossRef]

32. Gagniuc, P.A. Markov Chains: From Theory to Implementation and Experimentation; John Wiley \& Sons: Hoboken, NJ, USA, 2017.

33. Miller, C.E.; Tucker, A.W.; Zemlin, R.A. Integer Programming Formulation of Traveling Salesman Problems. J. ACM 1960, 7, 326-329. [CrossRef]

34. Gillet, B.; Miller, L. A Heuristic Algorithm for the Vehicle-Dispatch Problem. Oper. Res. 1974, 22, 340-349. [CrossRef]

35. Perboli, G.; Rosano, M.; Saint-Guillain, M.; Rizzo, P. Simulation-optimisation framework for City Logistics: An application on multimodal last-mile delivery. IET Intell. Transp. Syst. 2018, 12, 262-269. [CrossRef]

36. Perboli, G.; Rosano, M. Parcel delivery in urban areas: Opportunities and threats for the mix of traditional and green business models. Transp. Res. Part C 2019, 99, 19-36. [CrossRef] 